\title{
Medial Patellofemoral Ligament Reconstruction Following Total Knee Arthroplasty: A Case Report
}

\author{
Vishal Mehta, MD ${ }^{1}$ David Morawski, MD ${ }^{1}$ \\ ${ }^{1}$ Fox Valley Orthopedic Institute, Geneva, Illinois \\ J Knee Surg Rep 2016;2:e1-e3.
}

Address for correspondence Vishal Mehta, MD, Fox Valley Orthopedic Institute, 2525 Kaneville Road, Geneva, IL 60134 (e-mail: vmehta@fvortho.com).

\author{
Abstract \\ Keywords \\ - patella instability \\ - arthroplasty \\ - medial \\ patellofemoral \\ ligament
}

Patella instability is a rare but serious complication seen following total knee arthroplasty. The typical cause of patella instability in this setting is malpositioned components. While the mainstay of treatment is recognizing and correcting any malpositioning of the components, continued instability can remain and be a difficult problem to treat. Medial patellofemoral ligament (MPFL) reconstruction can be a helpful adjunct for the surgeon when faced with cases of continued instability. In this case report, we describe the successful use of MPFL reconstruction in conjunction with component revision for the treatment of postarthroplasty patella instability.
Total knee arthroplasty (TKA) is a commonly performed procedure that provides reliable pain relief to those suffering from knee arthritis. While technologies and surgical techniques have improved, complications can and do still occur. ${ }^{1-9}$ One of the most serious potential complications is postoperative patellar instability. ${ }^{4,10-12}$ Patellar instability can often be treated by correcting the rotation of malpositioned implants. When the implants are in appropriate position, this can be a more complex problem to treat. Recently, there has been interest in reconstructing the medial patellofemoral ligament (MPFL) to treat postoperative patellar instability. ${ }^{13,14}$ The purpose of this case report is to describe the use of MPFL reconstruction as an adjunct to femoral component revision and to treat such a case of postoperative patellar instability.

\section{Case Report}

A 65-year-old male presented to the clinic with left knee pain. He denied any episodes of trauma and described his pain as being located mostly at his medial compartment. His clinical examination revealed a small effusion and a range of motion from 10 to 115 degrees. He had a negative Lachman test and posterior drawer test and was stable to varus and valgus stress at 0 and 30 degrees. His patella tracked normally with some crepitus. There was no J sign and no lateral tilt of his patella on examination. His patella was neither subluxable nor dislocatable on examination and had a firm end point to lateral push. His radiographs were consistent with tricompartmental osteoarthritis (-Fig. 1). After failing conservative treatment options, he elected to proceed with a TKA.

The patient was taken to the operating room and a cruciate retaining TKA (Biomet, Warsaw, IN) was performed without complication. A subvastus approach was used and the patella resurfaced. The patient had a routine postoperative course until 6 weeks postoperatively when he began to complain of patella maltracking and pain after a fall. Clinical examination and radiographs revealed a laterally dislocated patella. The tibial and femoral components were carefully examined for evidence of malrotation, but no obvious malrotation was identified. A computed tomogaphy scan was also used to evaluate the rotational alignment of the components and revealed no evidence of malrotation. Treatment options were discussed and a release of the lateral retinaculum and medial reefing was performed. These were performed in a limited open fashion. The patient was placed in a knee immobilizer for 2 weeks following surgery and then began standard postarthroplasty physical therapy consisting of range of motion exercises, strengthening, and gait training. Upon discontinuation of the knee immobilizer, he was again found to have recurrent lateral patellar dislocations. Treatment options were again discussed and the patient decided to proceed with exploration of his knee arthroplasty with likely revision if malrotated components were found. In received

October 21, 2015

accepted after revision

July 2, 2016

published online

August 24, 2016
DOI http://dx.doi.org/

10.1055/s-0036-1587597. ISSN 2326-2729.
Copyright $\odot 2016$ by Thieme Medical Publishers, Inc., 333 Seventh Avenue, New York, NY 10001, USA. Tel: +1(212) 584-4662.
License terms

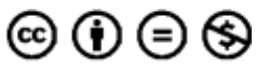




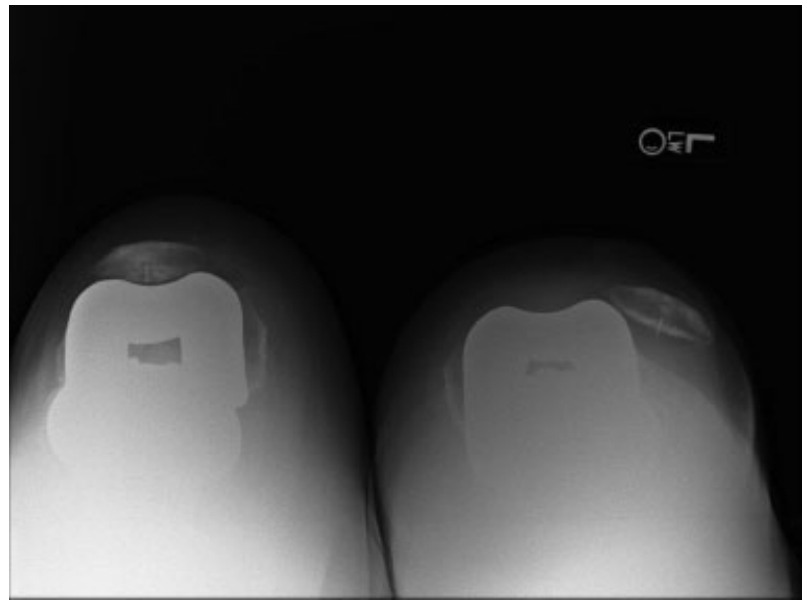

Fig. 1 Preoperative sunrise view.

addition, the decision was made to perform an MPFL reconstruction using a semitendinosus autograft, if deemed necessary, at the time of surgery.

The patient was taken back to the operating room and the arthroplasty was explored. The femoral component was examined in relation to the posterior condylar line and transepicondylar axis and found to be in neutral rotation. The component was revised to 3 degrees of external rotation and the knee taken through a range of motion. The tibial component was also evaluated and found to be positioned correctly with acceptable rotation using the tibial tubercle, the transmalleolar axis, and the second metatarsal as reference points. The patellar component itself was also evaluated and found to be well positioned on the medial aspect of the patella. Patella tracking was now evaluated by reapproximating the extensor mechanism and applying proximal tension through a flexion/ extension cycle. The patella continued to track laterally with every flexion/extension cycle. The medial retinacular tissue was found to be stretched and there was significant lateral retinacular tightness found. At this point, a lateral release was performed and the patella now remained in the trochlear groove throughout the flexion/extension cycle. However, the patella was easily dislocated with lateral push in the extended or near extended position. The decision was made to perform an MPFL reconstruction. This was done using a technique as described by Csintalan et al. ${ }^{15}$ The semitendinosus was identified at its insertion to the pes anserine. A closed ended tendon stripper was used to harvest it back to the musculotendinous junction. The tendon was doubled over and secured to the anatomic attachment of the MPFL adjacent to the medial epicondyle with a $7 \times 23 \mathrm{~mm}$ Bio-Tenodesis Screw System (Arthrex, Naples, FL). The two free limbs of the graft were shuttled between layers 2 and 3 of the retinaculum. A $4.5-\mathrm{mm}$ drill bit was used to make two drill holes in the superomedial aspect of the patella. The two free limbs of the graft were then brought through the drill holes, tensioned appropriately, and were sewn back to themselves securing the MPFL reconstruction ( - Fig. 2). This was performed with the knee in 15 degrees of flexion. The patella was tensioned to allow it to enter the trochlea smoothly during early flexion

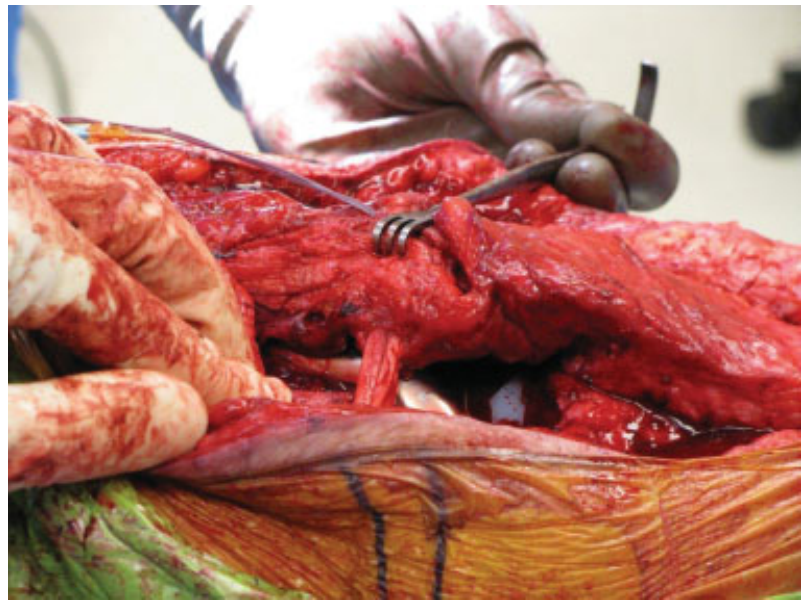

Fig. 2 Completed medial patellofemoral ligament reconstruction.

and also to provide a "check rein" to lateral dislocation. After the MPFL reconstruction and lateral release, the patella tracking was found to be greatly improved and the patella was no longer easily dislocated. Routine closure was performed and postoperative rehabilitation again consisted of immobilization in extension in a knee immobilizer for 2 weeks followed by routine postarthroplasty physical therapy.

The patient has had a routine postoperative course since his revision TKA and MPFL reconstruction. Postoperative radiographs reveal good tracking of his patellar component (-Fig. 3). He has had no further complaint of patellar maltracking or pain.

\section{Discussion}

Patellofemoral instability after TKA can be a very difficult problem to treat. It is often secondary to malposition of the components; therefore, revision surgery is usually

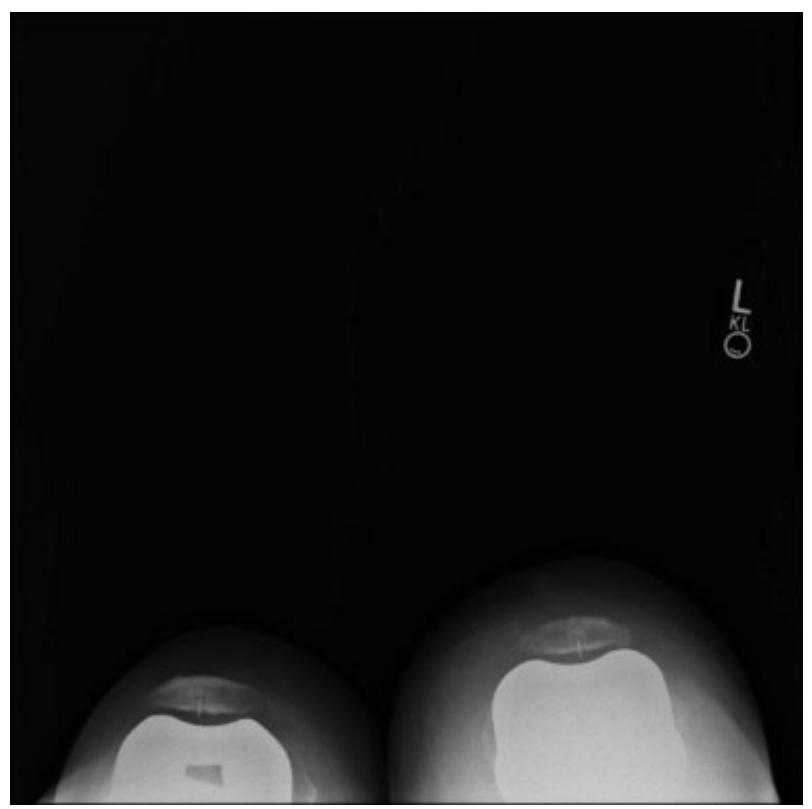

Fig. 3 Postoperative sunrise view. 
necessary. ${ }^{11,16,17}$ When the components are correctly positioned, an attempt at lateral release with or without proximal realignment may be considered. This technique has been previously described and good results have been reported by several authors. ${ }^{18,19}$ Distal realignment techniques have also been described with satisfactory results. These techniques involve a medial tibial tubercle osteotomy to change the Qangle of the patella and force it to track more medially. ${ }^{20}$ The exact role of MPFL reconstruction in the treatment pathways of postarthroplasty patellar instability remains to be defined. It seems reasonable to perform MPFL reconstruction as an adjunct or in addition to the previously mentioned realignment procedures. Most of the time, patella instability is secondary to component malposition. In cases of significant malposition, attempts to address only the soft tissue imbalances will likely fail. In these situations, careful and critical examination of the femoral, tibial, and patellar components is necessary. Revision surgery to return these components to the correct orientation will usually solve the problem. After the components have been restored to the correct orientation, there may still be a component of residual instability as a result of the previous patella dislocations. This is true in the case we have reported where we believe that there was likely a small component of femoral malrotation. After revising the femoral component, we chose to perform an MPFL reconstruction to reestablish the normal "check reign" to lateral patellar dislocation.

\section{Conclusion}

We demonstrate that an MPFL reconstruction may be a successful adjunct in managing patellar instability following TKA. We believe that this should be part of the armamentarium of the knee arthroplasty surgeon when encountering a patella that remains unstable or potentially unstable following treatment of any version or alignment issues. There are few reports in the literature regarding MPFL reconstruction in conjunction with TKA. ${ }^{13}$ We report the successful treatment of postarthroplasty patella dislocation with component revision and MPFL reconstruction.

\section{References}

1 Rand JA. Extensor mechanism complications after total knee arthroplasty. Instr Course Lect 2005;54:241-250
2 Farr J II, Barrett D. Optimizing patellofemoral arthroplasty. Knee 2008;15(5):339-347

3 Patel J, Ries MD, Bozic KJ. Extensor mechanism complications after total knee arthroplasty. Instr Course Lect 2008;57:283-294

4 Eisenhuth SA, Saleh KJ, Cui Q Clark CR, Brown TE. Patellofemoral instability after total knee arthroplasty. Clin Orthop Relat Res 2006;446(446):149-160

5 Kelly MA. Extensor mechanism complications in total knee arthroplasty. Instr Course Lect 2004;53:193-199

6 Hanssen AD, Pagnano MW. Revision of failed patellar components. Instr Course Lect 2004;53:201-206

7 Smith DE, McGraw RW, Taylor DC, Masri BA. Arterial complications and total knee arthroplasty. J Am Acad Orthop Surg 2001; 9(4):253-257

8 Berman AT, Schendel TE. Infected total knee arthroplasty: review. Orthopedics 1997;20(6):563-566

9 Huo MH, Sculco TP. Complications in primary total knee arthroplasty. Orthop Rev 1990;19(9):781-788

10 Anderson MJ, Becker DL, Kieckbusch T. Patellofemoral complications after posterior-stabilized total knee arthroplasty: a comparison of 2 different implant designs. J Arthroplasty 2002;17(4): 422-426

11 Kelly MA. Patellofemoral complications following total knee arthroplasty. Instr Course Lect 2001;50:403-407

12 Harwin SF. Patellofemoral complications in symmetrical total knee arthroplasty. J Arthroplasty 1998;13(7):753-762

13 Asada S, Akagi M, Mori S, Hamanishi C. Medial patellofemoral ligament reconstruction for recurrent patellar dislocation after total knee arthroplasty. J Orthop Sci 2008;13(3):255-258

14 Matsushita T, Kuroda R, Kubo S, Mizuno K, MatsumotoT, Kurosaka M. Total knee arthroplasty combined with medial patellofemoral ligament reconstruction for osteoarthritic knee with preoperative valgus deformity and chronic patellar dislocation. J Arthroplasty 2011;26(3):505.e17-505.e20

15 Csintalan RP, Latt LD, Fornalski S, Raiszadeh K, Inacio MC, Fithian DC. Medial patellofemoral ligament (MPFL) reconstruction for the treatment of patellofemoral instability. J Knee Surg 2014;27(2): 139-146

16 Malo M, Vince KG. The unstable patella after total knee arthroplasty: etiology, prevention, and management. J Am Acad Orthop Surg 2003;11(5):364-371

17 Briard JL, Hungerford DS. Patellofemoral instability in total knee arthroplasty. J Arthroplasty 1989;4(Suppl):S87-S97

18 Merkow RL, Soudry M, Insall JN. Patellar dislocation following total knee replacement. J Bone Joint Surg Am 1985;67(9): 1321-1327

19 Motsis EK, Paschos N, Pakos EE, Georgoulis AD. Review article: patellar instability after total knee arthroplasty. J Orthop Surg (Hong Kong) 2009;17(3):351-357

20 Price M, Malkani AL, Baker D. Fulkerson procedure for chronic patella component dislocation after total knee arthroplasty. J Arthroplasty 2009;24(6):914-917 